\title{
A Meshfree Solver for the MEG Forward Problem
}

\author{
Guido Ala ${ }^{1}$, Elisa Francomano ${ }^{2}$, Gregory E. Fasshauer ${ }^{3}$, Salvatore Ganci ${ }^{1}$, and Michael J. McCourt ${ }^{4}$ \\ ${ }^{1}$ DEIM, ${ }^{2}$ DICGIM, Università degli Studi di Palermo, Viale delle Scienze, 90128 Palermo, Italy \\ ${ }^{3}$ Department of Applied Mathematics, Illinois Institute of Technology, Chicago, IL 60616, USA \\ ${ }^{4}$ Department of Mathematical and Statistical Sciences, University of Colorado, Denver, CO 80202, USA
}

\begin{abstract}
Noninvasive estimation of brain activity via magnetoencephalography (MEG) involves an inverse problem whose solution requires an accurate and fast forward solver. To this end, we propose the Method of Fundamental Solutions (MFS) as a meshfree alternative to the Boundary Element Method (BEM). The solution of the MEG forward problem is obtained, via the Method of Particular Solutions (MPS), by numerically solving a boundary value problem for the electric scalar potential, derived from the quasi-stationary approximation of Maxwell's equations. The magnetic field is then computed by the Biot-Savart law. Numerical experiments have been carried out in a realistic single-shell head geometry. The proposed solver is compared with a state-of-the-art BEM solver. A good agreement and a reduced computational load show the attractiveness of the meshfree approach.
\end{abstract}

Index Terms-Biomagnetics, magnetoencephalography (MEG), method of fundamental solutions (MFS), meshfree methods.

\section{INTRODUCTION}

$\mathbf{T}$ HE PROBLEM of estimating the sources of neuronal activity in the human brain from electroencephalography (EEG) and magnetoencephalography (MEG) signals is of great interest both in clinical and basic health research. The brain activity generates small electric potential and magnetic field distributions that can be measured by means of an array of electrodes on the scalp, for EEG, or superconducting quantum interference devices (SQUID) located near the head, for MEG.

EEG can detect activity both in the sulci and at the top of the cortical gyri, whereas MEG is most sensitive to activity originating in sulci and provides a better spatial resolution [1].

Starting from a set of measured data (electric potential or magnetic fields), an inverse problem must be solved to estimate the corresponding neuronal activity sources. To this end, an accurate forward solver must be designed as a component in the solution of this inverse problem. Such a numerical tool computes the scalp potential and/or magnetic fields generated by a set of current sources representing the neural activity, given knowledge of both the physical properties of the biological tissues and the geometry of the head [2].

Here we focus the attention on the solution of the MEG forward problem. So far, the M/EEG forward problem has been addressed by traditional mesh-based numerical methods, whose literature is vast (see, e.g., [2] and references therein). Among these methods, the Boundary Element Method (BEM) has become the method of choice because of its efficiency with respect to the Finite Elements Method (FEM), and it is currently implemented in widely used software packages for M/EEG source analysis, e.g. FieldTrip [3]. However, the BEM involves costly numerical integration, requires an often nontrivial meshing of the domain boundaries at high quality and could potentially introduce mesh-related artifacts in the reconstructed neural activation pattern.

Manuscript received May 25, 2014; revised XXXX. Corresponding author: S. Ganci (email: salvatore.ganci@unipa.it).
Meshless methods have been previously proposed for solving the EEG [4] and the MEG [5] forward problem. However, they are domain methods, thus they may be outperformed by BEM from a computational efficiency standpoint.

\section{MEG ForWARD PROBLEM Formulation}

Common models rely upon a piecewise-constant conductivity approximation so that the head is described as a volume conductor composed of electrically homogeneous compartments. Compartment boundaries are extracted by means of anatomical MR or CT images. A detailed geometrical model with at least three compartments (brain, skull, scalp) is mandatory in solving the EEG forward problem, because electric potentials at the scalp are strongly distorted due to the conductivity difference between the tissues composing the head. In contrast, magnetic fields depend mostly on the electrical currents flowing in the high conductivity tissues, i.e., in the brain. A recent study [6] has shown, by means of a BEM approach, that the three nested compartment model performs best for the MEG forward problem. However a simple homogeneous model of the high-conductivity brain compartment - as proposed first in [7] and commonly adopted later on - provides acceptable accuracy. In this work, as a first step, the homogeneous single-shell model is considered.

Let $\Omega$ be the homogeneous domain that represents the brain, with boundary $\partial \Omega$ and electrical conductivity $\sigma$. The volume surrounding $\Omega$ can be considered as the ambient air, with negligible electrical conductivity. In light of the maximum significant frequency in bio-electromagnetic phenomena and of the characteristics of biological tissues, the MEG forward problem can be formulated by means of the quasi-stationary approximation of Maxwell's equations [1]. It is convenient to express the current density field at a point $\boldsymbol{p} \in \Omega$ as the sum of the source (impressed) current density $\mathbf{J}_{s}(\boldsymbol{p})$ and the volume current density, i.e.,

$$
\mathbf{J}(\boldsymbol{p})=\mathbf{J}_{s}(\boldsymbol{p})-\sigma \nabla \phi(\boldsymbol{p})
$$


where $\phi$ is the electric scalar potential. We shall concentrate our attention on the simplest case of a single neural source, representable by a current dipole of moment $\mathbf{Q}$ located at $\boldsymbol{p}^{\prime} \in$ $\Omega$ [1]. What follows can be extended to the case of many dipoles by simple application of the superposition principle.

With this position, the source current density is given by

$$
\mathbf{J}_{s}(\boldsymbol{p})=\mathbf{Q} \delta\left(\boldsymbol{p}-\boldsymbol{p}^{\prime}\right),
$$

where $\delta\left(\boldsymbol{p}-\boldsymbol{p}^{\prime}\right)$ is the Dirac delta function centered at the source point $\boldsymbol{p}^{\prime}$.

The solution of the MEG forward problem involves the solution of a potential problem on the boundary $\partial \Omega$. In fact the following equation holds:

$$
\nabla^{2} \mathbf{B}(\boldsymbol{p})=-\mu \nabla \times \mathbf{J}(\boldsymbol{p}),
$$

where $\mu$ is the magnetic permeability of the medium, supposed to be equal to that of air, and the current density on the righthand side is given by (1) if the electric scalar potential at $p$ is known. The solution of (3) under the assumption of a null magnetic field at an infinite distance from the sources, is given by the Biot-Savart law [8]:

$$
\mathbf{B}(\boldsymbol{p})=\frac{\mu}{4 \pi} \int_{\Omega} \mathbf{J}\left(\boldsymbol{p}^{*}\right) \times \frac{\boldsymbol{p}-\boldsymbol{p}^{*}}{\left\|\boldsymbol{p}-\boldsymbol{p}^{*}\right\|^{3}} \mathrm{~d} v\left(\boldsymbol{p}^{*}\right) .
$$

where $\mathrm{d} v$ is the differential volume element for $\Omega$.

By using (1), the integral above can be split into two parts by considering the contribution $\mathbf{B}_{s}(\boldsymbol{p})$ of the source current density, and the contribution of the volume current density:

$$
\mathbf{B}(\boldsymbol{p})=\mathbf{B}_{s}(\boldsymbol{p})-\frac{\mu}{4 \pi} \sigma \int_{\Omega} \nabla \phi\left(\boldsymbol{p}^{*}\right) \times \frac{\boldsymbol{p}-\boldsymbol{p}^{*}}{\left\|\boldsymbol{p}-\boldsymbol{p}^{*}\right\|^{3}} \mathrm{~d} v\left(\boldsymbol{p}^{*}\right) .
$$

For a dipole source, the following analytic expression of the first term $\mathbf{B}_{s}(\boldsymbol{p})$ is known [8]:

$$
\mathbf{B}_{s}(\boldsymbol{p})=\frac{\mu}{4 \pi} \mathbf{Q} \times \frac{\boldsymbol{p}-\boldsymbol{p}^{\prime}}{\left\|\boldsymbol{p}-\boldsymbol{p}^{\prime}\right\|^{3}} .
$$

The vector identity $\nabla \phi \times \nabla g=\nabla \times(\phi \nabla g)$, with $g=$ $\left\|\boldsymbol{p}-\boldsymbol{p}^{\prime}\right\|^{-1}$ and $\nabla g=\left(\boldsymbol{p}-\boldsymbol{p}^{\prime}\right)\left\|\boldsymbol{p}-\boldsymbol{p}^{\prime}\right\|^{-3}$, and the application of the following corollary of the Divergence Theorem:

$$
\int_{\Omega} \nabla \times \mathbf{F} \mathrm{d} v=\int_{\partial \Omega} \boldsymbol{n} \times \mathbf{F} \mathrm{d} s
$$

where $\mathbf{F}=\phi \nabla g, \boldsymbol{n}$ is the unit vector normal to the boundary and $\mathrm{d} s$ is the differential surface element for $\partial \Omega$, allow for the transformation of the volume integral over $\Omega$ in (5) into a surface integral over the boundary $\partial \Omega[9]$ :

$\mathbf{B}(\boldsymbol{p})=\mathbf{B}_{s}(\boldsymbol{p})-\frac{\mu}{4 \pi} \sigma \int_{\partial \Omega} \phi\left(\boldsymbol{p}^{*}\right) \boldsymbol{n}\left(\boldsymbol{p}^{*}\right) \times \frac{\boldsymbol{p}-\boldsymbol{p}^{*}}{\left\|\boldsymbol{p}-\boldsymbol{p}^{*}\right\|^{3}} \mathrm{~d} s\left(\boldsymbol{p}^{*}\right)$.

The electric scalar potential $\phi$ in $\Omega$ due to a current dipole is governed by a boundary value problem (BVP) for the Poisson equation [8]. In fact, by taking into account the continuity equation for $\mathbf{J}$, i.e.:

$$
\nabla \cdot\left(\mathbf{J}_{s}(\boldsymbol{p})-\sigma \nabla \phi(\boldsymbol{p})\right)=0
$$

the following BVP is obtained:

$$
\begin{cases}\sigma \nabla^{2} \phi(\boldsymbol{p})=\nabla \cdot \mathbf{J}_{s}(\boldsymbol{p}), & \boldsymbol{p} \in \Omega \\ \sigma \boldsymbol{n}(\boldsymbol{p}) \cdot \nabla \phi(\boldsymbol{p})=0, & \boldsymbol{p} \in \partial \Omega\end{cases}
$$

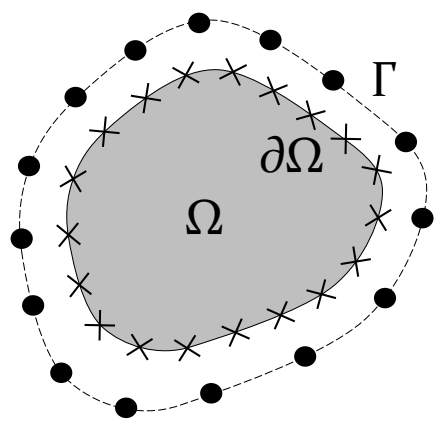

Fig. 1. Collocation points (crosses) and centers (dots) distributed on the physical boundary $\partial \Omega$ and on the fictitious boundary $\Gamma$, respectively.

Once $\phi$ is known on the boundary, the magnetic field at any point outside the head can be evaluated by (8).

\section{Methodology}

We propose the application of the Method of Fundamental Solutions (MFS) [10] via the Method of Particular Solutions (MPS) for solving the potential problem (10). The MFS approximates the solution $u$ of the given homogeneous BVP by a linear combination of fundamental solutions $K$ of the governing homogeneous PDE, i.e.,

$$
u(\boldsymbol{p}) \approx \sum_{\xi_{j} \in \Xi} c_{j} K\left(\boldsymbol{p}, \boldsymbol{\xi}_{j}\right), \quad \boldsymbol{p} \in \Omega,
$$

where $\Xi$ is a set of $M$ centers located on a fictitious boundary $\Gamma$ outside the physical domain $\Omega$ (Fig. 1) in order to avoid potential singularities of $K$ in the representation of the solution. The coefficients $c_{j}$ of the linear combination are determined by enforcing equality of $u(\boldsymbol{p})$ to the boundary conditions at a finite set of $N$ collocation points.

An inhomogeneous problem can be reduced to a homogeneous one by the MPS, i.e., by considering the solution $u$ as the sum of a particular solution $u_{p}$ and its associated homogeneous solution $u_{h}$.

The governing PDE of the scalar potential problem in $\Omega$ is a Poisson equation (see Section II). Let us express the scalar potential function in $\Omega$, by means of the MPS, as

$$
\phi(\boldsymbol{p})=\phi_{h}(\boldsymbol{p})+\phi_{p}(\boldsymbol{p}) .
$$

An analytical expression for a particular solution $\phi_{p}$ of the PDE of the BVP in $\Omega$, when a neural source is located at $\boldsymbol{p}^{\prime} \in \Omega$, is known [8]:

$$
\phi_{p}(\boldsymbol{p})=\frac{1}{4 \pi \sigma} \mathbf{Q} \cdot \frac{\boldsymbol{p}-\boldsymbol{p}^{\prime}}{\left\|\boldsymbol{p}-\boldsymbol{p}^{\prime}\right\|^{3}} .
$$

Therefore, the homogeneous term $\phi_{h}$ is given by the solution of the following BVP:

$$
\begin{cases}\nabla^{2} \phi_{h}(\boldsymbol{p})=0, & \boldsymbol{p} \in \Omega, \\ \boldsymbol{n}(\boldsymbol{p}) \cdot \nabla \phi_{h}(\boldsymbol{p})=-\boldsymbol{n}(\boldsymbol{p}) \cdot \nabla \phi_{p}(\boldsymbol{p}) & \boldsymbol{p} \in \partial \Omega .\end{cases}
$$

and it can be approximated, by means of the MFS, as a linear combination of fundamental solutions for the 3D Laplace equation, namely: $K(\boldsymbol{p}, \boldsymbol{q})=(4 \pi\|\boldsymbol{p}-\boldsymbol{q}\|)^{-1}$. The centers on the fictitious boundary can be picked by a simple procedure 
of deflation of the physical boundary with respect to its centroid. By introducing the MFS approximation, the solution is obtained by solving a linear system of size $N \times M$.

The only geometric quantities needed to compute the potentials are the normals to the boundary and the distances between the boundary collocation points and the centers; therefore, the proposed method is truly meshfree. Moreover, no costly numerical integration is needed and its implementation is straightforward.

It is worth mention that for certain problems and suitably smooth data and domains, the proposed method has been proved to be exponentially convergent [11], whereas the convergence rate of BEM and FEM is limited by the maximum degree of the polynomials adopted as basis functions.

\section{NumERICAL RESUlTS}

In order to assess the viability of the proposed approach in solving the MEG forward problem for a realistic single-shell head geometry, we have carried out a comparison with the BEM state-of-the-art formulation [12].

We consider the FieldTrip default subject anatomy. The inner skull surface is extracted by segmentation from the anatomical T1-weighted MRI of the subject's head. For BEM, the surface is described by a set of points connected by a triangle mesh. This mesh is constructed by projecting the vertices of an almost evenly triangulated sphere - whose center coincides with the centroid of the domain - onto the inner skull surface. Surface normals are straightforwardly derived from the triangle mesh. For MFS, a set of points is sufficient to describe the surface. For the sake of consistency with the BEM model, the collocation points on the surface are picked as the vertices of a triangle mesh that is constructed by the projection method described above for BEM, and the surface normals are evaluated by means of the same mesh. However, it is worth mention that surface normals can be estimated without forming a mesh by meshless methods for surface representation [13], [14].

The MFS linear system is solved in the least squares sense and a procedure of deflation of the physical boundary is adopted to locate the centers on the fictitious boundary. In particular we use a deflation coefficient equal to 1.3. This value of the deflation coefficient has been chosen empirically on the basis of numerical tests we carried out on spherical geometries for which the analytical solution of the potential problem is known [2].

The numerical experiments are conducted on a workstation equipped with a six core CPU Intel Xeon E5-2630 @ $2.30 \mathrm{GHz}$ and $24 \mathrm{~GB}$ of RAM.

First we compare the accuracy of the MFS solver and the BEM solver in evaluating the scalar potential on the inner skull surface. A single unitary dipole source is simulated in the brain (with electrical conductivity equal to $0.2 \mathrm{~S} / \mathrm{m}$ ) at roughly $1 \mathrm{~cm}$ from the inner skull surface. This choice is appropriate if one considers the location of the real neural sources in the cerebral cortex. Since there is no way of knowing the ground truth solution for realistic geometries, we choose a BEM solution obtained with a fine mesh (4500 triangles) as a reference.
The convergence to the reference solution is tested both for MFS and BEM. The relative 2-norm is adopted to estimate the accuracy with respect to the reference solution, as reported in Table I, where $N$ denotes the number of collocation points for MFS or the number of triangles for BEM. For the MFS solver the number of centers is half the number of collocation points.

TABLE I

PERFormanCE COMPARISON BETWEEN MFS AND BEM FOR THE POTENTIAL PROBLEM.

\begin{tabular}{ccccc}
\hline \hline \multirow{2}{*}{$N$} & \multicolumn{2}{c}{ MFS } & \multicolumn{2}{c}{ BEM } \\
\cline { 2 - 5 } & Accuracy & CPU Time $[\mathrm{s}]$ & Accuracy & CPU Time [s] \\
\hline 500 & 0.3269 & 0.101 & 0.5455 & 2.566 \\
1500 & 0.1137 & 0.299 & 0.1783 & 10.140 \\
2500 & 0.0930 & 1.189 & 0.1885 & 24.602 \\
3500 & 0.0821 & 2.756 & 0.1159 & 45.307 \\
4500 & 0.0585 & 5.585 & N/A & 74.508 \\
\hline \hline
\end{tabular}

The results shown in Table I suggest that the MFS solver outperforms the BEM solver. Moreover, the MFS provides a reduction in CPU time that becomes more significant as higher accuracy is requested, since no numerical integration is required in the assembly of the system matrix. Fig. 2 shows the potential maps obtained with BEM and with MFS at the finest discretizations.

Reusing the same real geometry, we now compare the computed external magnetic fields. In this case, we have assumed a set of 1000 unitary dipole sources randomly distributed inside the brain, with random orientations.

Fig. 3 shows the magnetic field maps on a SQUID helmet obtained with BEM and with MFS, respectively, at the finest discretizations. The considered magnetic field component is the one normal to the helmet surface. The maps depict a good agreement between the two solutions, with a 2-norm relative difference equal to 0.1220 .

\section{CONCLuSion}

In this paper we have shown that the solution of the MEG forward problem can be sought by the Method of Fundamental Solutions via the Method of Particular Solutions. The proposed method is a meshfree, boundary-type, integration-free and easy-to-implement alternative to mesh-based methods, such as the widely used Boundary Element Method.

We have successfully compared the proposed approach with the state-of-the-art BEM formulation in solving the MEG forward problem with a realistic single-shell head model. The method needs no meshing algorithms in the pre-processing stage - which simplifies the experimental setup - and no numerical quadrature to assemble the system matrix - which improves the computational load and is important when incorporating the forward solver within the solution of the inverse problem needed for identification of the neural sources.

Future work will be oriented to the application of the proposed approach to multi-compartment models. 


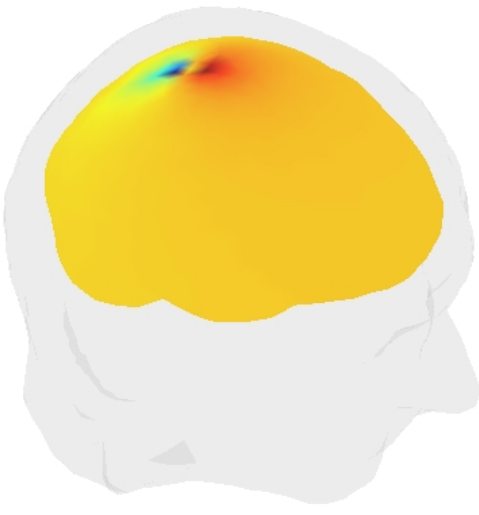

(a)

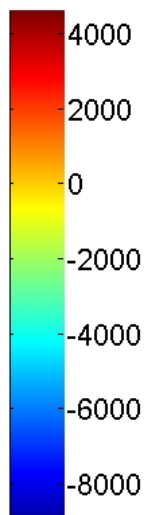

$-8000$

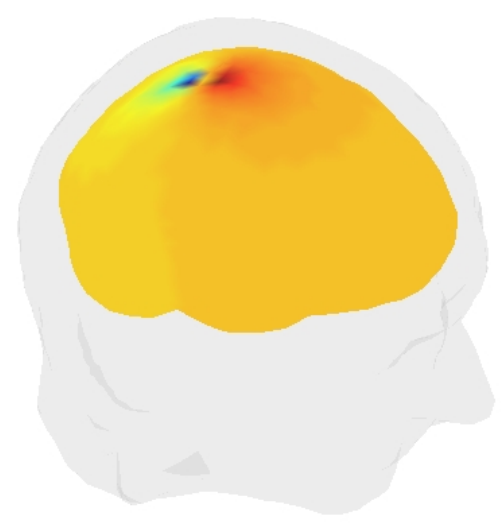

(b)

Fig. 2. Electric scalar potential [V] on the inner skull surface due to a unitary dipole calculated with (a) BEM and (b) MFS at the finest discretizations (BEM with 4500 triangles, MFS with 4500 collocation points and 2250 centers).

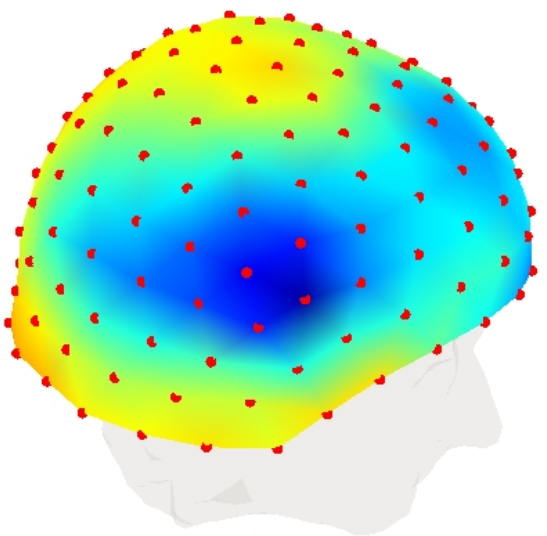

(a)
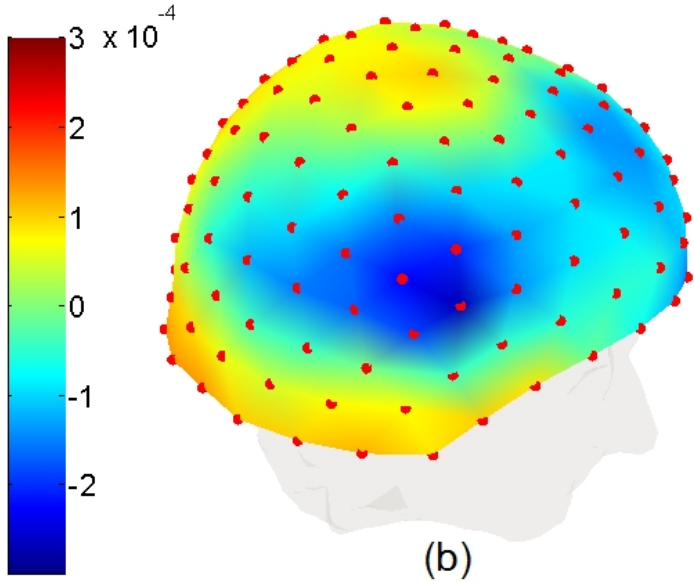

(b)

Fig. 3. Magnetic field [T] on the SQUID helmet (red dots as measurement points) due to 1,000 unitary dipoles randomly distributed inside the brain with random orientations, calculated with (a) BEM and (b) MFS at the finest discretizations (BEM with 4500 triangles, MFS with 4500 collocation points and 2250 centers)

\section{ACKNOWLEDGMENT}

This work was supported by University of Palermo, research projects FFR 2012-ATE-0440 and FFR 2012-ATE-0342.

\section{REFERENCES}

[1] M. Hämäläinen, R. Hari, R. Ilmoniemi, J. Knuutila, and O. Lounasmaa, "Magnetoencephalography - theory, instrumentation, and applications to noninvasive studies of the working human brain," Reviews of Modern Physics, vol. 65, no. 2, pp. 413-497, 1993.

[2] H. Hallez, B. Vanrumste, R. Grech, J. Muscat, W. De Clercq, A. Vergult, Y. D'Asseler, K. Camilleri, S. Fabri, S. Van Huffel, and I. Lemahieu, "Review on solving the forward problem in EEG source analysis," Journal of NeuroEngineering and Rehabilitation, vol. 4, no. 1, 2007.

[3] R. Oostenveld, P. Fries, E. Maris, and J. Schoffelen, "FieldTrip: open source software for advanced analysis of MEG, EEG, and invasive electrophysiological data," Computational intelligence and neuroscience, vol. 2011, p. 1, 2011.

[4] N. von Ellenrieder, C. Muravchik, and A. Nehorai, "A meshless method for solving the EEG forward problem," IEEE Transactions on Biomedical Engineering, vol. 52, no. 2, pp. 249-257, 2005.

[5] G. Ala, G. Di Blasi, and E. Francomano, "A numerical meshless particle method in solving the magnetoencephalography forward problem," International Journal of Numerical Modelling: Electronic Networks, Devices and Fields, vol. 25, no. 5-6, pp. 428-440, 2012.

[6] M. Stenroos, A. Hunold, and J. Haueisen, "Comparison of three-shell and simplified volume conductor models in magnetoencephalography," NeuroImage, vol. 94, pp. 337-348, 2014.
[7] M. Hämäläinen and J. Sarvas, "Realistic conductivity geometry model of the human head for interpretation of neuromagnetic data," IEEE Transactions on Biomedical Engineering, vol. 36, no. 2, pp. 165-171, 1989.

[8] J. Sarvas, "Basic mathematical and electromagnetic concepts of the biomagnetic inverse problem," Physics in Medicine and Biology, vol. 32, no. 1, p. 11, 1987.

[9] D. Geselowitz, "On the magnetic field generated outside an inhomogeneous volume conductor by internal current sources," IEEE Transactions on Magnetics, vol. 6, no. 2, pp. 346-347, 1970.

[10] G. Fairweather and A. Karageorghis, "The method of fundamental solutions for elliptic boundary value problems," Advances in Computational Mathematics, vol. 9, no. 1, pp. 69-95, 1998

[11] M. Katsurada and H. Okamoto, "The collocation points of the fundamental solution method for the potential problem," Computers \& Mathematics with Applications, vol. 31, no. 1, pp. 123-137, 1996.

[12] J. Kybic, M. Clerc, T. Abboud, O. Faugeras, R. Keriven, and T. Papadopoulo, "A common formalism for the integral formulations of the forward EEG problem," IEEE Transactions on Medical Imaging, vol. 24, no. 1, pp. 12-28, 2005.

[13] J. Carr, R. Beatson, J. Cherrie, T. Mitchell, W. Fright, B. McCallum, and T. Evans, "Reconstruction and representation of 3D objects with radial basis functions," in Proceedings of the 28th annual conference on Computer graphics and interactive techniques. ACM, 2001, pp. 67-76.

[14] G. Fasshauer, Meshfree Approximation Methods with MatLaB. River Edge, NJ, USA: World Scientific Publishing Co., Inc., 2007. 